Schweiz. Z. Tuberk. 1956;13:153-155

\title{
Le Dr Jean Morin (1890-1955)
}

Le 11 août 1955, le Dr Jean Morin s'est éteint dans sa propriété de Sombacour près de Neuchâtel.

II avait pris part, le 28 juin, à $\Gamma$ assemblée du Conseil de direction de l'Union Internationale contre la tuberculose et, au debut de juillet, comme cliaque année, il avait dirigé son Cours de Leysin. Rien ne faisait prévoir qu'une maladie inexorable allait $\Gamma$ emporter quatre semaines plus tard.

Cette disparition prématurée a profondément emu ceux qui, en Suisse et à $\Gamma$ étranger, connaissaient et estimaient ce grand médecin de notre pays.

Fils du Dr F. Morin qui avait été un des fondateurs de la Station climatérique de Leysin et, au debut du siècle, un des pionniers de la lutte antituberculeuse, Jean Morin s'était donné pour ideal de suivre les traces de son père.

Après avoir acquis une solide culture de médecine générale, au cours de ses stages hospitaliers à Lausanne où il fut interne et chef de clinique,

puis à Paris, Jean Morin se spécialisa en phtisiologie. En 1925, il prit la direction des sanatoriums populaíres de Leysin. Dès lors, toute sa vie fut vouée au traitement des malades pulmonaires et à la lutte contre la tuber-culose.

Doué d'une claire intelligence, d'un caractère ferme, d'une rare ca-pacité de travail et d'un sens exceptionnel de Torganisation, Jean Morin ne pouvait se contenter de tâches limitées, il était destine aux entreprises de grande envergure. II occupa rapidement, à Leysin, une place prépondérante et joua un role important au sein des organismes centraux de lutte antituberculeuse en Suisse et sur le plan international.

Médecin de sanatorium, il fut avant tout un thérapeute. Toujours à l'avant-garde du progrès dans le traitement de la tuberculose, il faisait bénéficier ses malades des techniques les plus modernes. $\mathrm{A}$ Гépoque où la collapsothérapie était notre principale arme de combat, il n'a cessé de préconiser le collapsus électif et économique, quel que soit le procédé utilise. II fut parmi les premiers à s'élever contre les thoracoplasties étendues inutilement mutilantes et à faire pratiquer des resections costales partielles limitées à la zone lésionnelle, ce qu'il aimait à appeler la «thoracoplastie topographique». II fut, au temps où la phrénicectomie jouissait d'une grande faveur, un des plus fervents adeptes de la méthode. Encourage par les résultats qu'il avait obtenus en pratiquant cette intervention à la place du pneumothorax chez des malades à plèvres libres, il avait pensé que la paralysie artificielle du nerf phrénique, comme operation autonome, était appelée à prendre une place importante parmi les autres procédés collapso-thérapiques. Ce fut le sujet de la these qui lui valut le titre de privat-docent à la Faculté de médecine de Lausanne en 1931.

Jean Morin n'avait cependant pas attendu ce grade universitaire pour exercer ses qualités pédagogiques. Dès 1929, suivant les traditions créées avant lui, à Leysin, par le Dr Burnand, il organisa, avec l'aide des médecins de la station, puis avec la collaboration des professeurs de la Faculté de Lausanne, des series de leçons sur la tuberculose qui attiraient chaque année à Leysin de nombreux étudiants. 
Mais ces cours n'étaient qu'une partie de son enseignement car, avec le don didactique qu'il possédait, il ne cessait de faire bénéficier ses assistants de sa vaste experience. Sous son impulsion, des etudes nombreuses et d'un grand intérêt furent faites et publiées par ses collaborateurs et ses élèves. II forma une phalange de phtisiologues dont les noms aujourd'hui sont connus.

II fut lui-même Гauteur d'une trentaine de publications fortement documentées, sur le traitement de la tuberculose, sur la tuberculose de Tenfant, etc., les plus récentes sur le Rimifon et le Marsilid.

Sur le plan plus general de la lutte antituberculeuse, Morin joua en Suisse un role très important. Au sein des comités dont il faisait partie, son jugement sûr, son esprit lucide, ses interventions toujours opportunes et concises lui valaient $\Gamma$ estime de chacun.

Membre du Comité de l'Association suisse contre la tuberculose depuis 1930, il faisait partie de son bureau dès 1946. En 1942, il avait été nommé membre du Conseil de direction de $\Gamma$ Union Internationale contre la tuberculose comme représentant de la Suisse.

Jean Morin participait à tous les congrès nationaux ou internationaux, concernant la tuberculose. II en fut à plusieurs reprises rapporteur. II ne manquait pas une seance, estimant que rien n'est indigne d'etre écouté et désirant toujours être complètement informé. Tout ce qu'il entendait s'ordonnait aussitôt dans son esprit et sa mémoire était infaillible.

Jean Morin n'était pas un théoricien. III ne se perdait pas en discussions académiques. III ne s'attardait pas aux problèmes épineux qui se dé-battent aux limites du connu et qu'il laissait aux savants et aux homines de laboratoire. Essentiellement pratique, il préférait la solidité de quelques dogmes consacrés ou provisoirement admis comme valables. Par temperament, il lui fallait une doctrine. La théorie de Ranke dont il était un ardent défenseur, le satisfaisait pleinement. II n'aimait pas le doute; la certitude semblait lui être un confort nécessaire. C'était là, probablement, une attitude de facade, mais qui donnait à sa personnalité cette carrure si particulière.

Dans le domaine thérapeutique, il poursuivait ses experiences avec méthode; le schématisme de ses conclusions pouvait déconcerter. Toute-fois, s'il lui fallait faire marche arrière, il faisait preuve d'une grande souplesse d'esprit. II Ta prouvé au cours de ces dernières années, en appliquant largement dans les établissements qu'il dirigeait la medication anti-bacillaire et en jugeant avec une rare objectivité les résultats qu'il en avait obtenus. Conscient des profonds changements d'oríentation que devait provoquer le traitement niédicamenteux de la tuberculose, Jean Morin a été un des premiers médecins de sanatorium à affïrmer sa foi en ces remèdes nouveaux et à préconiser un réajustement de notre front de combat contre la tuberculose. Ce bouleversement, auquel beaucoup ne peuvent se ré-signer, lui était apparu, par delà les intérêts particuliers menaces, comme le résultat heureux et réconfortant d'une lutte à laquelle son père et lui avaient, pendant un demi-siècle, consacré leur existence. Peu avant sa mort, il m'avait exprimé sa joie et son espoir de voir la tuberculose à la veille d'etre vaincue. La carrière de Jean Morin se sera done achevée dans cette lueur d'aurore.

Dr M. Gilbert 